\title{
Transient photocurrents as a spatially resolved probe of carrier transport and defect distributions in silicon thin films
}

\author{
Steve Reynolds ${ }^{\mathrm{a}, *}$, Rudi Brüggemann ${ }^{\mathrm{b}}$, Björn Grootoonk ${ }^{\mathrm{c}}$, Vlad Smirnov ${ }^{\mathrm{c}}$ \\ a School of Engineering, Physics and Mathematics, University of Dundee, Nethergate, Dundee DD1 4HN, Scotland, United Kingdom \\ b Carl von Ossietzsky Universität Oldenburg, Institut für Physik, D-26111 Oldenburg, Federal Republic of Germany \\ ' Institut für Energie- und Klimaforschung (IEK-5 Photovoltaik), Forschungszentrum Jülich, Leo Brandt Strasse, D-52425 Jülich, Federal Republic of Germany
}

\section{A R T I C L E I N F O}

\section{Article history:}

Received 3 July 2012

Received in revised form

18 September 2012

Accepted 11 October 2012

Available online 12 November 2012

\section{Keywords:}

Amorphous silicon

Microcrystalline silicon

Defects

Photoconductivity

\begin{abstract}
A B S T R A C T
Transient photocurrent spectroscopy (TPC) yields the energetic distribution of localised states in disordered semiconductors from an analysis of the decay of photocurrent with time following a short laser pulse. By comparing results at different laser excitation wavelengths, and hence absorption depths, information on spatial non-uniformities may also be inferred. Here we investigate the use of TPC as a spatial probe with reference to two thin-film silicon systems; amorphous silicon subjected to various lightinduced degradation regimes, and microcrystalline silicon grown on a range of 'seed' layers. Computer simulation is used to support experimental findings, and to identify sensitivity and resolution limitations.
\end{abstract}

(c) 2012 Elsevier B.V. All rights reserved.

\section{Introduction}

Transient photocurrent spectroscopy (TPC) is a sensitive probe of the energetic distribution of band-tail and defect states in disordered semiconductors, which control key material parameters including carrier mobility and lifetime. The technique is based on inversion of the decay in photocurrent $I(t)$ following a nanosecond flash of laser light incident on a gap-cell under voltage bias, to yield the density of localised states (DOS) in terms of a multiple-trapping transport model [1,2].

We have previously shown that a degree of spatial sensitivity may also be obtained using TPC [3-5]. This variant, which we refer to here as S-TPC, utilises the differing absorption depths of the light flash with wavelength to generate carriers either uniformly, or preferentially at one or other surface. For example, in an amorphous $(\mathrm{a}-\mathrm{Si}: \mathrm{H})$ or microcrystalline $(\mu \mathrm{c}-\mathrm{Si}: \mathrm{H})$ silicon film $1 \mu \mathrm{m}$ thick, carrier generation by red light is fairly uniform, but green light generates a much higher carrier concentration close to the incident surface. It was thus proposed that the resulting $I(t)$ will be linked to the DOS in this region. Similar claims have been made when using other photoconductivity techniques including steady state [6], constant-photocurrent method [7] and modulated photocurrent spectroscopy [8].

\footnotetext{
* Corresponding author. Tel.: +44 1382 384559; fax: +44 1382384389.

E-mail address: s.z.reynolds@dundee.ac.uk (S. Reynolds).
}

To investigate S-TPC experimentally, a film with a known, controllable, spatial distribution of defects is required. Working towards this goal, we have compared TPC decays obtained from an amorphous silicon film in a thermally annealed state, and after prolonged exposure to white light, and white light band-pass filtered with a peak transmittance at $520 \mathrm{~nm}$ (green). As light-soaking increases the density of dangling bonds in proportion to the carrier generation rate [9], it is anticipated that a variable (but at best semi-quantitative) spatial defect profile may be realised in this way.

Microcrystalline silicon films are substantially more stable to light-induced degradation, but given their inhomogeneous structure evolution during growth [10] electronic transport properties are also likely to be spatially non-uniform. Here we apply S-TPC to a series of microcrystalline films with thin 'seed' layers deposited on the glass substrate prior to the main film growth. Such layers influence the structure evolution, leading to a compact film with more uniform crystalline content and improved solar cell performance [11]. Investigation of electronic transport and defect distributions in such state-of-the-art material is thus both timely and of high relevance. The crystalline volume fraction was measured using Raman spectroscopy, using both red and blue exciting lines to enable variations in crystallinity between bulk and surface to be estimated [12].

Although photo-carriers will initially interact with localised states in the immediate vicinity, concentration gradients will inevitably lead to diffusion into the bulk of the film and, given the asymmetry of electron and hole transport, the subsequent 
spatial evolution with time is likely to be complex. The degree of success of the S-TPC method will depend upon these detailed interactions. To identify the key features we have carried out computer simulations in two-layer composite films with amorphous silicon-like DOS profiles, in which the defect density of a thin 'surface' layer is varied. By integration of the current components in each layer parallel to the film we obtain the measured $I(t)$ which is then processed to yield a DOS for comparison with the model input.

\section{Experimental}

Amorphous silicon films were prepared in a commercial 13.56 MHz PECVD reactor (InterSolar Ltd.) with a silane:hydrogen process gas concentration (SC) of $95 \%$, chamber pressure $0.7 \mathrm{mbar}$ and substrate temperature $200^{\circ} \mathrm{C}$. The film thickness was $1.7 \mu \mathrm{m}$. Chromium contacts $5 \mathrm{~mm}$ in length separated by $1 \mathrm{~mm}$ defined the gap cell. White light-soaking of amorphous films (referred to as WLS) was carried out using an ELH lamp providing $1000 \mathrm{~W} / \mathrm{m}^{2}$ at the sample. For green light-soaking (GLS) the same arrangement was used, but with a Spiers-Robertson BG18 band-pass filter placed in front of the sample. Each light-soaking session was $15 \mathrm{~h}$ in duration. Thermal annealing (TA) was carried out as required, in air at $180^{\circ} \mathrm{C}$ for $2 \mathrm{~h}$

Microcrystalline silicon samples were prepared at IEK-5, Forschungszentrum Jülich by PECVD at $94.7 \mathrm{MHz}$ with a power of 10 watts, gas pressure of 0.4 mbar and substrate temperature of $180^{\circ} \mathrm{C}[13]$. A layer approximately $50 \mathrm{~nm}$ thick was firstly deposited on to a Corning 7059 glass substrate, roughened to improve film adhesion. The crystallinity of this 'seed' layer was adjusted by setting the silane concentration $\mathrm{SC}_{\text {Seed }}$ between $2 \%$ and $5.3 \%$ in a series of five depositions. Each sample was completed by deposition at a gas ratio of $5.3 \%$ to give a total film thickness of $1 \mu \mathrm{m}$. The degree of crystallinity was estimated using Raman spectroscopy [12] in terms of the ratio of the integrated intensity of the crystalline bands to the total intensity (amorphous plus crystalline), referred to as $I_{\text {CRs. }}$. The blue $(488 \mathrm{~nm})$ exciting line has an absorption depth of around $200 \mathrm{~nm}$ and gives an indication of the crystallinity towards the free surface of the film, whereas the red $(647 \mathrm{~nm})$ exciting line has an absorption depth of some $3 \mu \mathrm{m}$ and hence will return a value averaged over the entire film thickness. $5 \mathrm{~mm} \times 5 \mathrm{~mm}$ silver contact pads $0.5 \mathrm{~mm}$ apart were deposited on the top of the film to form a gap-cell.

In the TPC experiment [14], nanosecond light pulses were generated by a Laser Science VSL337 $\mathrm{N}_{2}$ laser plus dye attachment. Wavelengths of $510 \mathrm{~nm}$ and $640 \mathrm{~nm}$ were selected by insertion of the appropriate dye cuvette. These are referred to hereafter as GL (green laser) and RL (red laser), respectively. The pulses were attenuated to give a total flux of $10^{11}$ photons $\mathrm{cm}^{-2}$ at the sample, corresponding to a photocarrier density of order $10^{15} \mathrm{~cm}^{-3}$.

When light-soaking, and also when performing the TPC experiment, either side of the sample could be illuminated by rotating the sample holder. The sides of the sample illuminated in a given experiment are referred to hereafter as contact side (CS) (the film/air interface) and substrate side (SS) (the film/glass interface).

All TPC measurements were made at room temperature with an applied voltage bias of $100-300$ volts. Following appropriate preamplification, current transients from $1 \mathrm{~ns}$ to $10 \mathrm{~s}$ were recorded on a Tektronix TDS3052 digital storage oscilloscope. Averaging of successive transients reduced noise to an acceptable level. Data were transferred to a PC and processed into a single logarithmically spaced file.

The DOS was extracted by firstly applying a discrete Fourier transform to the $I(t)$ data, to obtain the ac current $I(\omega)$ where $\omega$ is the angular frequency. Within a multiple-trapping formalism, $I(\omega)$ may then be mapped directly to the DOS via the simple approximate relationship [15]:

$\operatorname{DOS}(\omega) \propto \frac{\sin (\phi(\omega))}{|I(\omega)|}$

where $\phi(\omega)$ is the phase angle of the photocurrent relative to the excitation. More usefully, the DOS may be plotted against energy (relative to the conduction band mobility edge) by noting that

$E=k T \ln (\nu / \omega)$

where $k$ is Boltzmann's constant, $T$ the absolute temperature and $v$ the attempt-to-escape frequency. This approach enables an arbitrary DOS to be extracted from TPC data with a resolution of order $k T$.

Computer simulation of film parameters was carried out using the SC-Simul program developed at the University of Oldenburg [16]. This is a one-dimensional numerical simulation which solves the Poisson equation, the continuity equations for electrons and holes, and the current transport equations including drift, diffusion, and thermionic emission over barriers, if present, in the valence and conduction bands. Further details are given in Ref. [16].

The model structure consisted of three parallel layers in contact: (i) a $100 \mathrm{~nm}$ layer of a-Si:H representing the surface region, (ii) a $900 \mathrm{~nm}$ layer of a-Si:H representing the bulk film, (iii) a $1 \mathrm{~mm}$ insulator restricting current flow normal to the film. Spatially uniform sets of localised states (exponential band tails and amphoteric dangling bonds) were specified in layers (i) and (ii). In this study only defect densities were varied. Conduction and valence band tail slopes of 30 and $50 \mathrm{meV}$, respectively, and a Gaussian defect distribution of width $0.15 \mathrm{eV}$ centred at $0.9 \mathrm{eV}$ below the valence band edge with a correlation energy of $0.25 \mathrm{eV}$ were used throughout, all other parameters being maintained at default values. The SC-Simul program is designed primarily to simulate currents normal to the plane of the film, in structures such as solar cells, and a post-simulation numerical integration of the carrier density in each slice of the film parallel to the substrate (typically $10 \mathrm{~nm}$ thick) was performed to calculate the co-planar current. The DOS was extracted from the simulated $I(t)$ curve in an identical manner to the experiment.

\section{Results and discussion}

\subsection{S-TPC of amorphous silicon films}

Fig. 1(a) and (b) shows the $I(t)$ decays and resulting DOS for the $\mathrm{a}-\mathrm{Si}: \mathrm{H}$ sample following light soaking with white light from the contact side of the film. The annealed state curves are shown for comparison.

In the case of materials with band tails and deep defects, such as silicon thin films, the $I(t)$ decay in the ns to s range generally consists of [1]: (i) a region of constant slope at the beginning of the observable range $\left(10^{-9}-10^{-8} \mathrm{~s}\right)$ due to carrier thermalisation in the band-tail; (ii) a steeper fall in current due to carrier trapping into deep defects; (iii) a complex combination of trapping and emission involving deeper tail states and defects; (iv) a sharp fall at the onset of free carrier loss (recombination). It is seen in Fig. 1(a) that the main difference in the $I(t)$ curves is associated with the onset of region (ii) (carrier trapping into deep defects). This occurs at progressively shorter times with increasing deep defect density.

Thus in common with previous studies of disordered silicon films and devices using TPC, including polysilicon thin-film transistors [17] and amorphous silicon sensors [18], we find the photocurrent to be dispersive, and the DOS to be comprised of band-tails and defects. The band-tails appear to be unaffected by light-soaking, but the defect density is significantly modified. The 

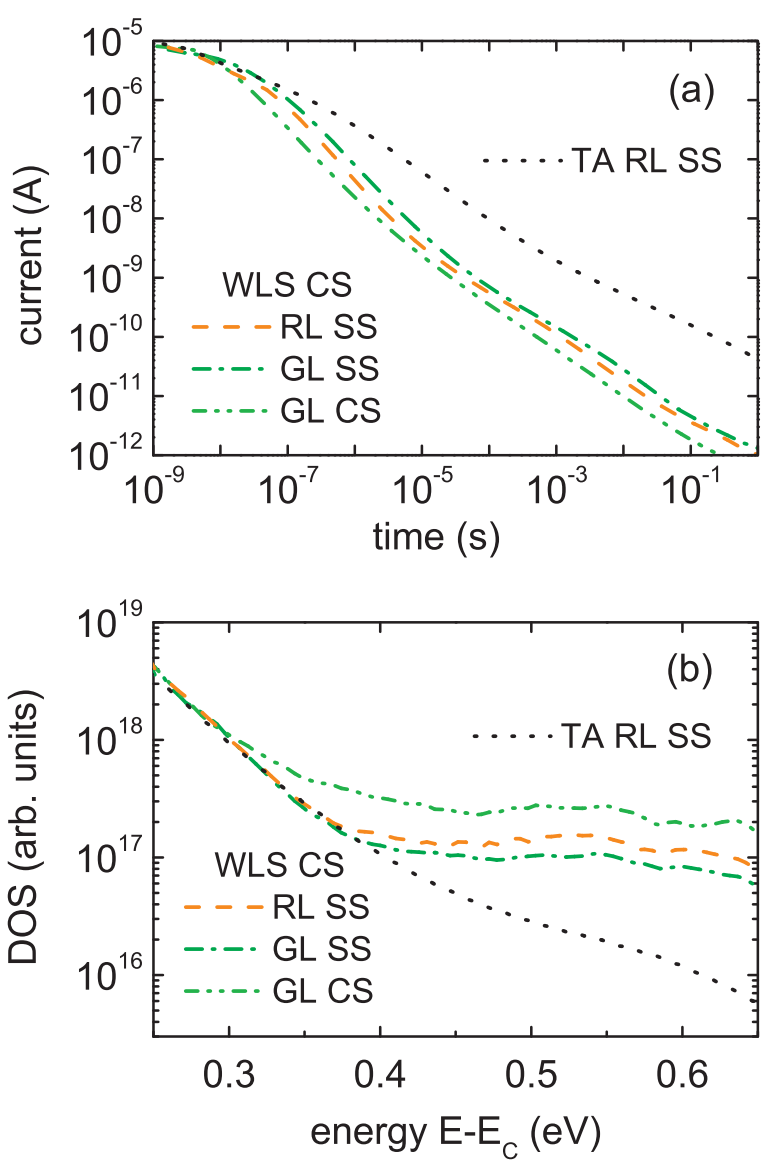

Fig. 1. (a) Experimental TPC decay plotted on log-log scale, (b) TPC DOS plotted on log-linear scale, for a-Si:H film white light-soaked from contact side, obtained using red and green laser pulses incident from substrate and contact sides as indicated. Curves for annealed film, obtained using red laser incident from substrate side, are shown for comparison. An attempt-to-escape frequency of $10^{12} \mathrm{~s}^{-1}$ was used to scale the energy axis. DOS scales are normalised at $0.3 \mathrm{eV}$. (For interpretation of the references to color in this figure legend, the reader is referred to the web version of the article.)

defects are manifested as a plateau in the DOS curve, and exhibit no sharp features.

During light soaking, the shorter wavelength fraction of white light results in an increased generation rate at the contact side, with the longer wavelength fraction penetrating further into the bulk. If the defect profile is assumed to follow the generation rate profile then we anticipate a higher density of defects at the contact side, set against a more uniform background, as illustrated in Fig. 2. On comparison with the result from the annealed sample, S-TPC clearly shows that light-soaking increases the DOS at energies deeper than $0.4 \mathrm{eV}$ below the conduction band edge, which is where dangling bond defects are expected to occur. The highest DOS is registered using a green laser pulse incident from the contact side, as anticipated since the green pulse is fully absorbed in the region of higher defect density following light-soaking (Fig. 2). The DOS obtained using a red laser pulse, which is more uniformly absorbed and thus, we propose, samples the average defect density, is a factor of 2-3 smaller than this. The above-bandgap fraction of the white light spectrum is almost completely absorbed by the $1.7 \mu \mathrm{m}$ film. Thus few light-induced defects are expected to be generated at the substrate side - the average generation rate in a $100 \mathrm{~nm}$ layer in this region is estimated to be around $1 \%$ of that in an equivalent layer at the contact side. However, the DOS registered using a green light pulse from the substrate side indicates a defect density only a factor of 4 less than at the contact side, which is a factor of 5-10 higher

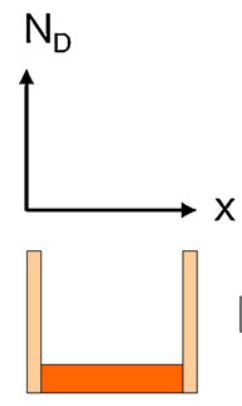

(i)

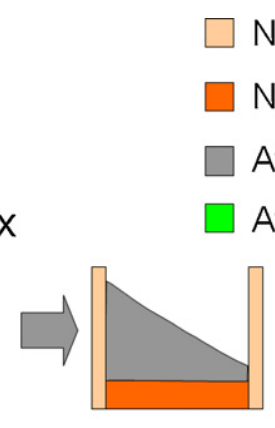

(ii)
Native surface defects

Native bulk defects

After white LS

After green LS

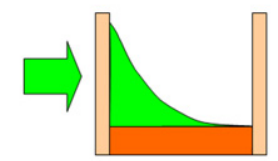

(iii)
Fig. 2. Suggested spatial defect distribution in amorphous silicon film: (i) after annealing, (ii) after white light-soaking, (iii) after green light-soaking. Arrows indicate direction of incident light. (For interpretation of the references to color in this figure legend, the reader is referred to the web version of the article.)

than the intrinsic defect density following annealing. Previously we showed that a contribution arises from intrinsic surface defects [4], as included in Fig. 2, but this does not account for more than a small fraction. It seems more likely that this larger than anticipated DOS value highlights a limitation of S-TPC, namely the migration of photocarriers generated by the pulse into the film as time proceeds. The extent to which this affects the apparent DOS will depend on how far a typical carrier migrates before it is trapped at a defect. Capture time is inversely proportional to defect density [1], and so carriers generated in a region of low defect density are more likely to have migrated into a region of higher defect density before being trapped at a defect. The trapping time into defects is manifested clearly in the $I(t)$ curves in Fig. 1(a) as a fall in current between 10 and $100 \mathrm{~ns}$. Carrier migration will be more rapid if band tails are steep, leading to higher values of effective mobility and diffusion constant.

It should be noted that a similar, although reversed, set of results are obtained when light-soaking from the substrate side (not presented here). As the electrostatic environments of the film surfaces (air and glass) are quite different, this observation suggests that surface band-bending has at most a minor effect.

Fig. 3 shows the DOS for the a-Si:H sample following lightsoaking with green light, this time from the substrate side. The defect distribution is envisaged to follow the profile illustrated in Fig. 2. As with white light soaking, the apparent DOS measured

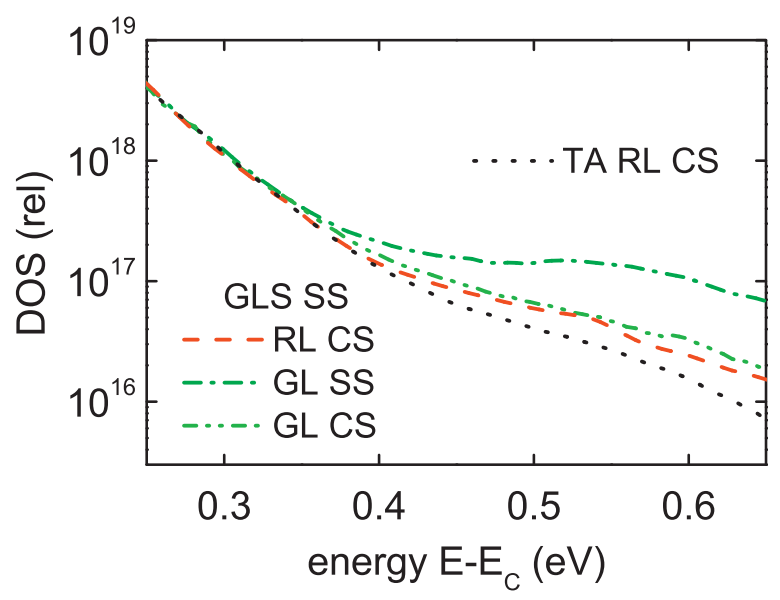

Fig. 3. Experimental TPC DOS plots on log-linear scale for a-Si:H film green lightsoaked from substrate side, obtained using red and green laser pulses incident from substrate and contact sides as indicated. Curves for annealed film, obtained using red laser incident from contact side, are shown for comparison. An attempt-to-escape frequency of $10^{12} \mathrm{~s}^{-1}$ was used to scale the energy axis. DOS scales are normalised at $0.3 \mathrm{eV}$. (For interpretation of the references to color in this figure legend, the reader is referred to the web version of the article.) 


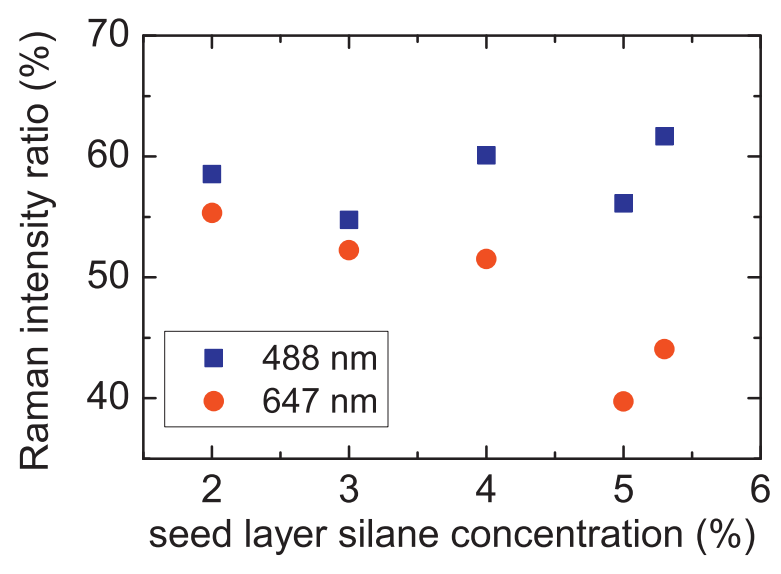

Fig. 4. Raman intensity ratio $I_{\mathrm{CRS}}$ vs. seed layer silane concentration $\mathrm{SC}_{\text {Seed }}$ for series of microcrystalline silicon films prepared on roughened substrates. Laser excitation is incident from the contact side of the film in each case. (For interpretation of the references to color in the text, the reader is referred to the web version of the article.)

using TPC with a green pulse from the side where the light-soaking source is applied is around a factor of 3 larger than from the opposite side. However, the DOS measured using a red pulse is only a factor of 2 larger than the native defect density. This indicates a low average light-induced defect density in the bulk when compared with white light-soaking, consistent with the reduced fraction of less strongly absorbed light.

Finally, we note that no strong difference in TPC decays was observed by increasing the thermal annealing time above $1 \mathrm{~h}$. It appears that following annealing the sample is returned more or less to its initial defect distribution, of native surface and bulk defects, irrespective of previous light-soakings.

\subsection{S-TPC of microcrystalline silicon films}

The Raman data shown in Fig. 4 indicates that the crystalline volume fraction of the top surface of the film (blue excitation) remains approximately constant at $55-60 \%$, independent of $\mathrm{SC}_{\text {seed }}$ over the range under consideration. The average value (red excitation) is similar to that of the top surface for $\mathrm{SC}_{\text {seed }}$ values of $2 \%$ and $3 \%$, but can be seen to fall when $\mathrm{SC}_{\text {seed }}$ is increased above $4 \%$. This is indicative of more compact crystalline bulk film growth initiated by the pre-deposition of a highly crystalline seed layer [11]. A cartoon of the growth profiles envisaged is shown in Fig. 5.

The TPC results shown in Fig. 6 are taken on a film with a $2 \%$ $\mathrm{SC}_{\text {seed }}$ pre-deposition. There are clear differences in the DOS curves depending on the laser wavelength and the side from which the pulse is incident. The results suggest the highest density of deep

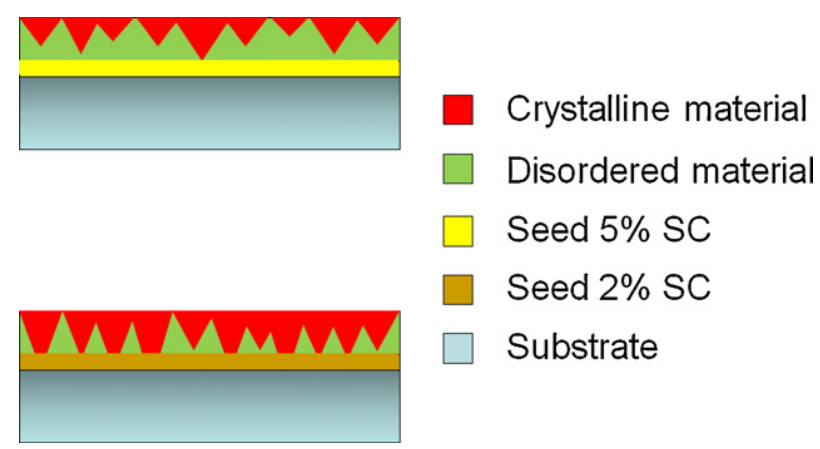

Fig. 5. Influence of seed layer on film growth (schematic), inferred from Raman measurements in Fig. 4 and electron micrographs (not presented here). (For interpretation of the references to color in the text, the reader is referred to the web version of the article.)

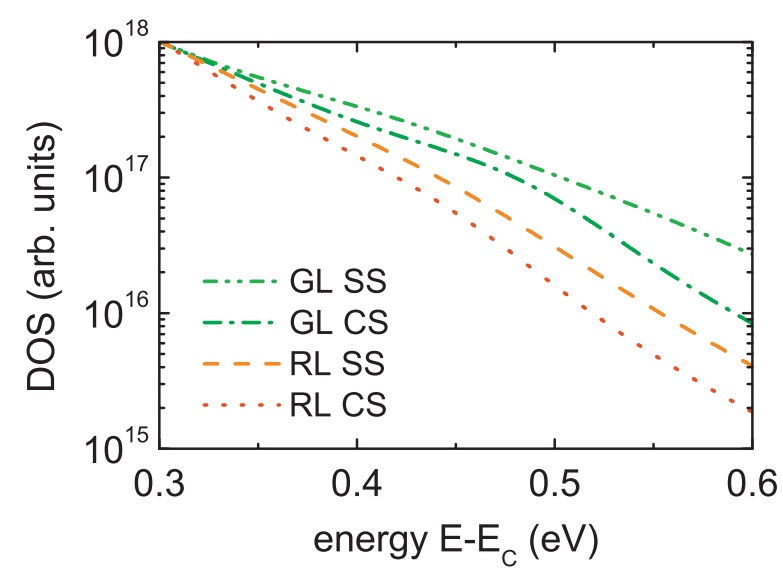

Fig. 6. Experimental TPC DOS plots on log-linear scale for $\mu \mathrm{c}-\mathrm{Si}: \mathrm{H}$ film prepared with a seed layer silane concentration of $2 \%$, obtained using red and green laser pulses incident from substrate and contact sides as indicated. An attempt-to-escape frequency of $10^{12} \mathrm{~s}^{-1}$ was used to scale the energy axis. DOS scales are normalised at $0.3 \mathrm{eV}$. (For interpretation of the references to color in this figure legend, the reader is referred to the web version of the article.)

states occurs in the vicinity of the seed layer (green laser, applied to substrate side). This is in keeping with the ESR work of Astakhov et al. [19] who showed an increase of around 1 order of magnitude in the spin density for highly crystalline films, when compared with the optimum phase mixture. The next highest defect density is recorded at the top surface of the film (green laser, contact side), where more uniform crystalline growth is fully established. Bearing in mind our earlier work on amorphous silicon films [5], there may be an additional contribution from surface states. The results obtained using a red laser pulse lie substantially below those for the green laser, which suggests that for this sample the average defect density, representative of the bulk of the film, is lower than at either surface. The red pulse incident from the substrate side indicates a higher DOS than from the contact side, which might be anticipated since in this case the red light intensity is higher in the region of the seed layer compared with the top surface, and so more initial photo-current will be generated there. However on examining in detail the data obtained with the red laser across the seed series (not presented here), no clear pattern emerges.

Fig. 7(a)-(e) shows the variation in TPC DOS measured with the green laser pulse from either side of the film, as $\mathrm{SC}_{\text {seed }}$ is varied. At values below $4 \%$, the DOS at the substrate side of the film lies above that at the contact side, at $4 \%$ the DOS is similar in both cases, and above $4 \%$ the situation is reversed. This supports the trend identified in the previous section.

However, caution should be exercised when interpreting TPC signals in microcrystalline silicon films. Earlier TPC work suggests that the photocurrent decay for films prepared over a range of SC exhibits complex behaviour that is difficult to interpret consistently solely in terms of the DOS [20]. Shifts in Fermi level position due to inadvertent doping and/or the presence of adsorbates [21] may result in changes in occupancy of the DOS, rather than changes in the DOS itself. At present therefore we cannot be certain whether defect densities associated with higher- or lower crystallinity material solely control the S-TPC decay.

\subsection{Computer simulations}

Simulations based on a model in which the $100 \mathrm{~nm}$ surface layer defect density $N_{\mathrm{D} 1}$ is varied between $3 \times 10^{15} \mathrm{~cm}^{-3}$ and $1 \times 10^{17} \mathrm{~cm}^{-3}$, with the $900 \mathrm{~nm}$ bulk layer defect density $N_{\mathrm{D} 2}$ fixed at $3 \times 10^{15} \mathrm{~cm}^{-3}$, are shown in Fig. 8(a) and (b). As with the experimental data, the simulated TPC curve exhibits behaviour associated 


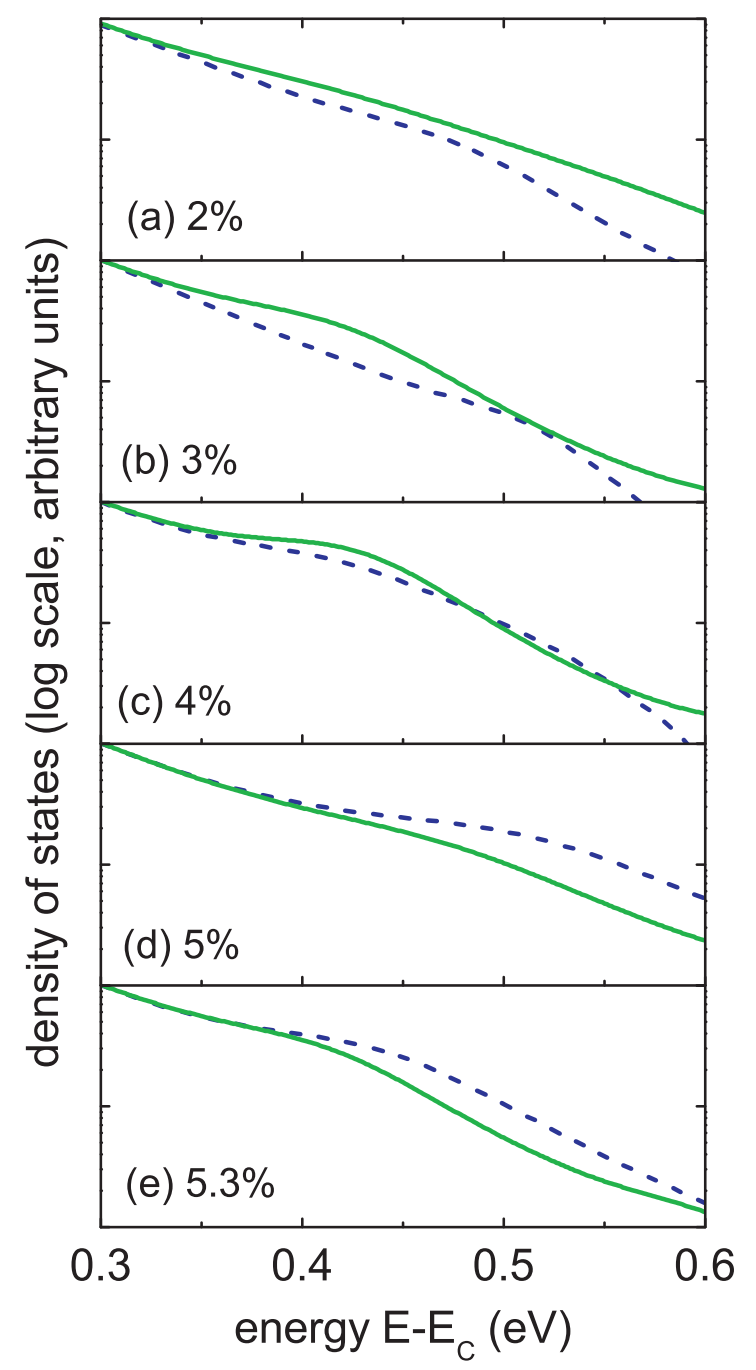

Fig. 7. Experimental TPC DOS plots on log-linear scale for $\mu \mathrm{c}-\mathrm{Si}: \mathrm{H}$ films prepared over the indicated range of $\mathrm{SC}_{\text {seed }}(\mathrm{a})$-(e), obtained using green laser pulses incident from substrate (solid line) and contact (dashed line) sides. An attempt-to-escape frequency of $10^{12} \mathrm{~s}^{-1}$ was used to scale the energy axis. DOS scales are normalised at $0.3 \mathrm{eV}$. (For interpretation of the references to color in this figure legend, the reader is referred to the web version of the article.)

with the physics outlined at the beginning of Section 3.1. The laser pulse wavelength is constant throughout at $500 \mathrm{~nm}$. A plot of the DOS obtained from a uniform layer $\left(N_{\mathrm{D} 1}, N_{\mathrm{D} 2}=1 \times 10^{17} \mathrm{~cm}^{-3}\right)$ is shown for comparison. It can be seen that while the band tail and general profile of the defect distribution is reproduced, the recovered density is somewhat below the model input value, by a factor of 4 at $N_{\mathrm{D} 1}=1 \times 10^{17} \mathrm{~cm}^{-3}$. In Fig. 9, the effect of varying the wavelength of the laser pulse with constant $N_{\mathrm{D} 1}=1 \times 10^{17} \mathrm{~cm}^{-3}$ is shown. Where the absorption depth is less than the layer 1 thickness, so that carrier generation occurs primarily in this layer, the recovered DOS profile converges to the limit described above. When the absorption depth is greater than the entire film thickness, carrier generation is quite uniform and the DOS converges to a lower value, close to the weighted mean. Overall then, the simulation results support the view that a degree of spatial resolution is possible, but they also convey a more pessimistic message concerning the ability to recover the absolute DOS in such cases. A closer look at the simulated spatial distribution of free carrier density with time confirms that carrier migration between the surface layer and the bulk is a significant effect, and will be reported on in detail in a later publication. There are a number of factors that influence the
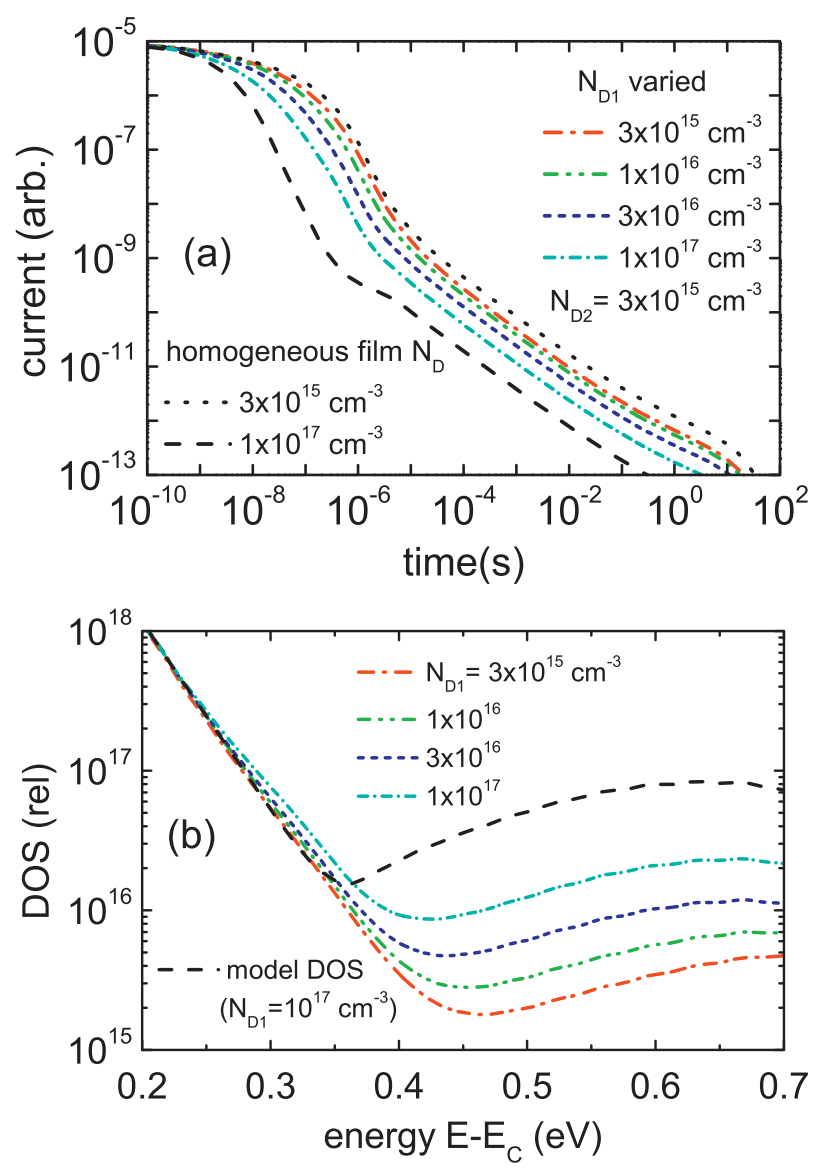

Fig. 8. (a) Simulated TPC decay plotted on log-log scale for a-Si:H composite film model, vs. surface layer defect density $N_{\mathrm{D} 1} .500 \mathrm{~nm}$ laser pulse incident on surface layer. Bulk layer defect density $N_{\mathrm{D} 2}$ is held constant at $3 \times 10^{15} \mathrm{~cm}^{-3}$. Two additional simulations, for homogeneous films at the ends of the range with uniformly absorbed laser pulses, are shown for comparison. (b) DOS curves recovered from data in (a). The model DOS input for $N_{\mathrm{D} 1}=1 \times 10^{17} \mathrm{~cm}^{-3}$ is shown as a guide.

extent to which the $I(t)$ curve under strongly absorbed light bears the imprint of the surface region of the film. Viewed in terms of our simplified structural model there are two films electrically in parallel, and the influence of one may only be separated successfully from the other if its contribution to the photocurrent is dominant over the experimental timescale, which in practice seems unlikely.

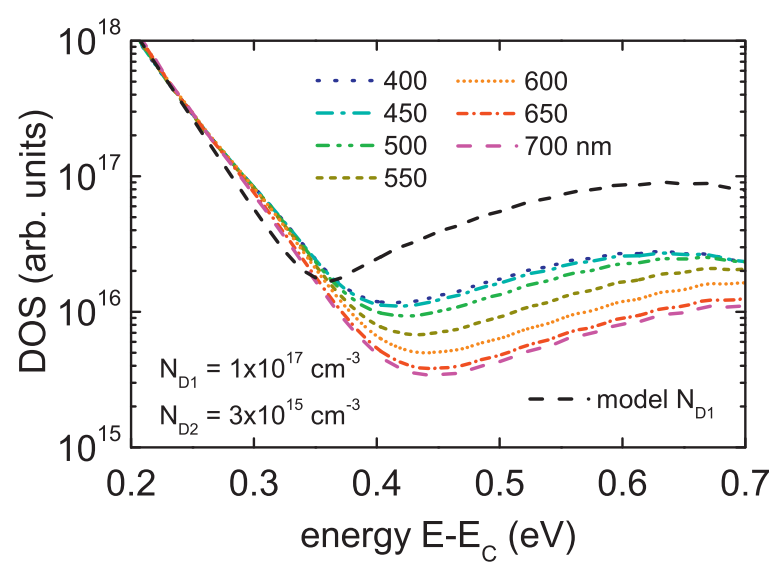

Fig. 9. Simulated TPC DOS plots on log-linear scale for a-Si:H composite film model, vs. laser pulse wavelength. Laser incident on surface layer. Bulk and surface layer defect densities held constant at the values indicated. The model DOS input for $N_{\text {D1 }}=1 \times 10^{17} \mathrm{~cm}^{-3}$ is shown for comparison. 


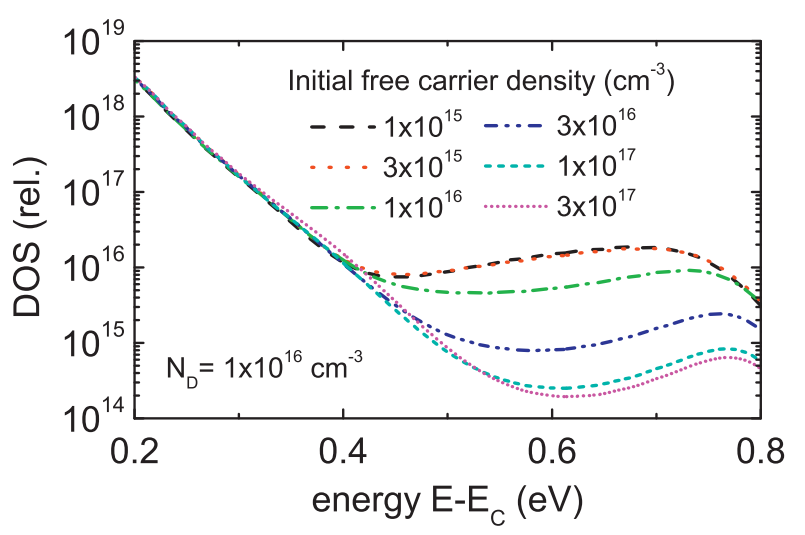

Fig. 10. Simulated TPC DOS plots on log-linear scale for a-Si:H homogeneous film model, vs. initial free carrier density (uniform throughout film). Progressive saturation of defects with increasing free carrier density is clearly visible once it exceeds the defect density.

It was also noted that since in S-TPC carrier generation is confined to a thin layer, the local free carrier density prior to deep trapping may be sufficiently large to cause trap filling and saturation, leading to a reduction and distortion of the apparent DOS, as illustrated in Fig. 10. If the generation rate is reduced to compensate, the photocurrent will be reduced in proportion and the signal to noise ratio in the TPC experiment will be degraded.

\section{Conclusions}

The apparent density of defect states in silicon thin films measured by transient photocurrent spectroscopy is dependent upon the wavelength of the light pulse used to generate photocarriers, and on the side from which the pulse is incident. In the case of light-soaked amorphous silicon films, these effects are consistent with an asymmetric spatial distribution of lightinduced defects, whereas in microcrystalline silicon the asymmetry results from differing structure compositions, mediated by a controllable 'seed' layer pre-deposition step. Spatially resolved TPC has been investigated by computer simulation, which predicts a degree of spatial sensitivity. Although the defect energy is correctly returned the apparent DOS magnitude may significantly underestimate the model value. This shortfall is associated with migration of carriers out of the surface layer prior to the occurrence of deep trapping. The model enables a more detailed study to be made of the limitations, and potential range of application, of the technique.
Future work might usefully include simulating the rather simpler steady-state system (photocurrent vs. wavelength) studied by Ghosh and Ganguly [6]. While this approach may not yield energyresolved information on defects, provided recombination occurs via defects located within the generation envelope the photocurrent magnitude should correlate with the defect density, enabling spatial resolution to be obtained.

\section{Acknowledgements}

The authors thank Markus Hülsbeck and Andreas Lambertz for their technical assistance, and Friedhelm Finger for his helpful comments and support of this collaboration.

\section{References}

[1] C. Main, MRS Symposium Proceedings Series 467 (1997) 167.

[2] C. Main, Journal of Non-Crystalline Solids 299-302 (2002) 525.

[3] R. Brüggemann, C. Main, S. Reynolds, Journal of Physics: Condensed Matter 14 (2002) 6909.

[4] S. Reynolds, C. Main, R. Brüggemann, Materials Research Society Symposium Proceedings 762 (2003) 137.

[5] S. Reynolds, C. Main, R. Brüggemann, Journal of Materials Science - Materials in Electronics 14 (2003) 615.

[6] S. Ghosh, G. Ganguly, Journal of Applied Physics 68 (1990) 5896

[7] P. Kounavis, D. Mataras, N. Spiliopoulos, D. Rapacoulias, Proceedings of 12th EU-PVSEC, Amsterdam, 11-15 April, 1994, p. 144

[8] J.P. Kleider, C. Longeaud, P. Roca, I. Cabarrocas, Journal of Applied Physics 72 (1992) 4727

[9] M. Stutzmann, W.B. Jackson, C.C. Tsai, Physical Review B32 (1985) 23.

[10] L. Houben, M. Luysberg, P. Hapke, R. Carius, F. Finger, H. Wagner, Philosophical Magazine A 77 (1998) 1447.

[11] V. Smirnov, C. Das, T. Melle, A. Lambertz, M. Hulsbeck, R. Carius, F. Finger Materials Science and Engineering B 159 (2009) 44.

[12] C. Ross, Y. Mai, R. Carius, F. Finger, Progress in Photovoltaics: Research Applications 19 (2011) 715.

[13] O. Vetterl, F. Finger, R. Carius, P. Hapke, L. Houben, O. Kluth, A. Lambertz, A Mück, B. Rech, H. Wagner, Solar Energy Materials and Solar Cells 62 (2000) 97.

[14] S. Reynolds, C. Main, D.P. Webb, M.J. Rose, Philosophical Magazine B 80 (2000) 547.

[15] C. Main, R. Brüggemann, D.P. Webb, S. Reynolds, Solid State Communications 83 (1992) 401.

[16] M. Rösch, PhD Thesis, University of Oldenburg, 2003, Available at: http://oops.uni-oldenburg.de/frontdoor.php?source_opus=277. The SC-Simul software may be downloaded from http://www.greco.uni-oldenburg.de/ 26446.html

[17] R. Pandya, B.A. Khan, Journal of Applied Physics 62 (1987) 3244.

[18] N. Wyrsch, G. Choong, C. Miazza, C. Ballif, Sensors 8 (2008) 4656.

[19] O. Astakhov, R. Carius, F. Finger, Yu. Petrusenko, V. Borysenko, D. Barankov, Physical Review B79 (2009) 104205.

[20] S. Reynolds, V. Smirnov, C. Main, R. Carius, F. Finger, MRS Symposium Proceedings 715 (2002) 345.

[21] S. Reynolds, V. Smirnov, F. Finger, C. Main, R. Carius, Journal of Optoelectronics and Advanced Materials 7 (2005) 91. 